\title{
Multiple Sclerosis Misdiagnosis: A Persistent Problem to Solve
}

\author{
Maria I. Gaitán* and Jorge Correale \\ Neurology Department, Fundación para la Lucha contra las Enfermedades Neurológicas de la Infancia (FLENI), Buenos Aires, \\ Argentina
}

Keywords: multiple sclerosis, misdiagnosis, McDonald criteria, diagnostic error, multiple sclerosis mimics

\section{OPEN ACCESS}

Edited by:

Björn Tackenberg,

University of Marburg, Germany

Reviewed by:

Klemens Ruprecht,

Charité Medical University of Berlin,

Germany

Felix Luessi,

Johannes Gutenberg University

Mainz, Germany

*Correspondence:

María I. Gaitán

migaitan@fleni.org.ar:

minesgaitan@gmail.com

Specialty section:

This article was submitted to

Multiple Sclerosis and

Neuroimmunology,

a section of the journal

Frontiers in Neurology

Received: 29 January 2019

Accepted: 17 April 2019

Published: 07 May 2019

Citation:

Gaitán MI and Correale J (2019)

Multiple Sclerosis Misdiagnosis: A

Persistent Problem to Solve.

Front. Neurol. 10:466

doi: 10.3389/fneur.2019.00466
Despite significant refinement in multiple sclerosis (MS) diagnosis in recent decades, no specific disease biomarker exists, as a result of which, confirming the diagnosis is not always a straightforward process. MS has heterogeneous clinical and imaging manifestations, which not only differ between patients, but also vary in individual patients over time. Disease signs and symptoms, presence of oligoclonal bands (OCB) and MRI findings have limited specificity, and misdiagnosis remains a problem with significant clinical and psychosocial implications for both patients as well as health care providers. Although the problem of MS misdiagnosis is known, true incidence and prevalence is not. Some data is available from case reports, and recent publications from specialized MS centers reported around 30\% of cases originally referred for MS were finally diagnosed with other diseases (1). One study conducted in four academic MS centers revealed over 50\% of patients carried a misdiagnosis for at least 3 years, 70\% had received disease-modifying therapy (DMTs), and $31 \%$ experienced unnecessary morbidity as a direct result (2). A wide range of conditions can be mistaken for MS, including: migraine, cerebral small vessel disease, fibromyalgia, functional neurological disorders, and neuromyelitis optica spectrum disorders, along with uncommon inflammatory, infectious and metabolic conditions $(1,3)$. As early initiation of DMTs improves short- and long-term clinical outcome, pressure to establish timely diagnosis may also increase risk of misdiagnosis. Interestingly, revision of the McDonald criteria in 2017 emphasizes the problem of misdiagnosis risk in clinical practice (4). The McDonald criteria, first introduced for MS diagnosis in 2001, with revisions made in 2005, 2010, and 2017, incorporated MRI to fulfilled the criteria of dissemination in time (DIT) and dissemination in space (DIS), increasing early diagnosis sensitivity. However, McDonald criteria were not developed to distinguish MS from other conditions, but to identify MS or high likelihood of disease in patients with a typical clinical isolated syndrome (CIS) (i.e., optic neuritis, transverse myelitis, brainstem syndrome) (4).

In this sense, wrong application of the clinical criteria may: (1) include misinterpretation of a clinical symptom that is not caused by inflammatory demyelination as a demyelinating event, (2) acceptance of a historical episode of neurologic dysfunction, in the absence of contemporaneous or current objective evidence (neurologic examination, evoked potentials, or MRI) providing corroboration, and (3) overreliance on MRI abnormalities in patients with not specific symptoms (2).

Initial reports of MS misdiagnosis began at the end of the 1980s, interestingly, if MRI had been available, diagnostic error would have been prevented in most cases (5). However, when MRI became widely used, overestimations of radiological findings started to be reported (6). Despite introduction of radiological criteria for DIT and space DIS for both MS and for CIS (7), the problem has remained unsolved. A previous study showed that patients which were referred to MS centers for exclusive imaging findings suggestive of MS, they mostly did not have MS; most brain MRI T2 lesions were due to: migraine, age-related diseases, or hypertension (8). In more recent work, MRI contributors to MS misdiagnosis included: (1) overreliance on imaging abnormalities corresponding to DIS in patients with "non-specific neurologic symptoms," (2) incorrect interpretation of a subcortical lesion as periventricular or juxtacortical to meet DIS criteria, and (3) misinterpretation of DIT due to variations in MRI slice orientation (2). 
Many factors can help perpetuate misdiagnosis. For example: not reconsidering an established diagnosis even in the presence of certain atypical clinical or para-clinical features, so-called "red flags," or incomplete evidence of MS. In this regard, many MS specialists after encountering atypical MS syndromes on several occasions, may consider them as part of the spectrum of disease; when patients are referred to a new clinician for non-medical reasons, the physician usually accept their preestablished diagnosis (9); also, contrary to what one may believe, not all patients are relieved to discover they do not have MS, and may experience resistance to a new diagnosis (10); last, neurologists not willing to admit medical errors or experiencing diagnostic uncertainty may also be a contributing factor.

MS misdiagnosis may increase morbidity as a result of psychological damage, risk associated with DMTs and corticosteroids use, inadequate treatment $(2,3)$, worsening of underlying disease such as in neuromyelitis optica spectrum disorders (NMOSDs; (11), or delay in treatment of other potentially curable pathologies. Misdiagnosed patients are also sometimes included in clinical trials, which can confuse trial results and expose patients to inappropriate treatment. Other important point to consider is the economic impact of treating misdiagnosed patients, particularly in developing countries.

Work is needed to prevent misdiagnosis; neurologists must look out for clinical findings or diagnostic test results raising red flags. Three main factors could help avoid misdiagnosis. First a correct McDonald criteria application, looking for typical clinical syndromes, or historical events backed by objective corroborative evidence. Second, an appropriate application of radiological McDonald criteria: correct classification of lesion topography (juxtacortical, periventricular, infratentorial, spinal cord), juxtacortical lesiones are next to the cortex, and periventricular lesions are situated around ventricles. Use of the central vein sign in susceptibility-weighted images, may play a role in discriminating MS from its radiological mimics $(12,13)$. MS diagnosis should not be based solely on MRI findings. In difficult cases, a second opinion from a neuro-radiologist is recommended. Finally, in cases with inconclusive diagnosis, presentation of a syndrome other than a typical CIS, or insufficient clinical or MRI evidence to support diagnosis, CSF and serum OCB assay should be performed (4). Isoelectric focusing and immunoblotting or immunofixation for IgG, significantly increase diagnostic sensitivity (95-100\%) and specificity (86-87\%) (14) (Table 1). However, it is important to remember that OCBs are not specific to MS. Conversely, findings atypical for MS in CSF suggest other diagnoses (22). In negative cases, an alternative diagnosis should be considered. Cervical MRI, in search of asymptomatic spinal cord lesions, should be run in these patients (23).

Because patients with MS and NMOSDs have overlapping features care should be taken in this differential diagnosis, particularly in certain population such as African American, Asian, Latin American, and pediatric patients. Most patients with NMOSDs have detectable serum antibodies that target the water channel aquoporin-4 (NMO-IgG), which facilitates an early diagnostic distinction between patients who have NMOSDs and those who have MS $(17,24)$. However, around $30 \%$ of patients presenting with features of NMOSDs are AQP4seronegative, some of them may have antibodies reactive with myelin oligodendrocyte glycoprotein (MOG) (18). Nevertheless, the diagnostic sensitivity and specificity of anti-MOG antibody have not been fully validated. Because treatments of MS and

TABLE 1 | Recommendations to prevent MS misdiagnosis.

\section{When Applying 2017 McDonald criteria:}

The McDonald criteria were developed to diagnose MS or a high likelihood of the disease in subjects with a typical CIS after other diagnoses were excluded.

Consider to postpone definitive diagnosis of MS when a categorical typical CIS is not present. Further clinical and radiological follow-up is recommended before a definitive diagnosis.

Do not consider a previous event in the absence of current or contemporaneous objective evidence providing corroboration.

A neurologist with experience in MS should integrate the clinical case with complementary studies in order to make a solid diagnosis.

When clinical and brain MRI evidences are not enough for MS diagnosis, in patients with a progressive course at onset, in children, older individuals, and/or non-white populations, additional workout is needed including spinal cord MRI or CSF examination.

When atypical clinical syndromes and abnormal MRI findings:

Consider alternative diagnosis, common causes of misdiagnosis: migraine, fibromyalgia, conversion or psychogenic disorders, MRI changes due to vascular disease, NMOSDs

When discriminating MS from NMOSD and anti-MOG disease: Consider the following aspects.

\begin{tabular}{|c|c|c|c|}
\hline & MS & NMOSDs & Anti-MOG \\
\hline OCB & $95-100 \%(14)$ & $22 \%(15)$ & $6-13 \%(16)$ \\
\hline Anti-AQP4 (Ab) & Negative & $70 \%(17)$ & Negative \\
\hline Anti-MOG (Ab) & Negative & Negative & Positive (18) \\
\hline EBV-Seroprevalence* & $>99 \%($ anti-EBNA-1, anti-EBNA-2, anti-VCA) & $52 \%$ (anti-EA) (19) & 9\% (anti-VCA) (20) \\
\hline MRZ (21) & $78 \%$ & $1-2 \%$ & $1-2 \%$ \\
\hline
\end{tabular}

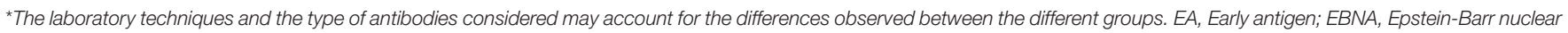
antigen; VCA, Viral capside antigen. 
NMOSDs are different, and disease modifying treatments for MS can induce severe exacerbations serological testing for AQP4 and MOG should be done in patients with features suggestive of NMOSDs, particularly in populations at high risk (25) (Table 1).

Recently, the detection of a polyspecific intrathecal humoral immune response against measles, rubella and varicela zoster virus called MRZ reaction has been shown to be a highly specific marker of MS, but only moderately sensitive (21). Interestingly, MRZ reaction was absent in virtually all patients with NMOSDs and anti-MOG antibody associated CNS demyelination analyzed so far $(21,26)$. However, it must be taken into consideration that, the clinical reliability of MRZ reaction in a given population may be influenced by the natural prevalence and the local vaccination coverage against these three viruses (Table 1).

Given that Epstein-Barr virus (EBV) seroprevalence in patients with MS is practically universal (27), EBV seronegative persons with symptoms suggestive of CIS/MS are not likely to be frequently identified (28). It is conceivable that in those cases, EBV seronegativity could represent a clinically useful biomarker for a diagnosis other than CIS/MS $(29,30)$ (Table 1).

\section{REFERENCES}

1. Yamout BI, Khoury SJ, Ayyoubi N, Doumiati H, Fakhreddine M, Ahmed SF, et al. Alternative diagnoses in patients referred to specialized centers for suspected MS. Mult Scler Relat Disord. (2017) 18:85-9. doi: 10.1016/j.msard.2017.09.016

2. Solomon AJ, Bourdette DN, Cross AH, Applebee A, Skidd PM, Howard DB, et al. The contemporary spectrum of multiple sclerosis misdiagnosis: a multicenter study. Neurology. (2016) 87:1393-9. doi: 10.1212/WNL.0000000000003152

3. Solomon AJ, Klein EP, Bourdette D. "Undiagnosing" multiple sclerosis: the challenge of misdiagnosis in MS. Neurology. (2012) 78:1986-91. doi: 10.1212/WNL.0b013e318259e1b2

4. Thompson AJ, Banwell BL, Barkhof F, Carroll WM, Coetzee T, Comi G, et al. Diagnosis of multiple sclerosis: 2017 revisions of the McDonald criteria. Lancet Neurol. (2018) 17:162-73. doi: 10.1016/S1474-4422(17)30470-2

5. Rudick RA, Schiffer RB, Schwetz KM, Herndon RM. Multiple sclerosis. The problem of incorrect diagnosis. Arch Neurol. (1986) 43:578-83.

6. Poser CM. Misdiagnosis of multiple sclerosis and beta-interferon. Lancet. (1997) 349:1916. doi: 10.1016/S0140-6736(05)63920-7

7. McDonald WI, Compston A, Edan G, Goodkin D, Hartung HP, Lublin FD, et al. Recommended diagnostic criteria for multiple sclerosis: guidelines from the International Panel on the diagnosis of multiple sclerosis. Ann Neurol. (2001) 50:121-7. doi: 10.1002/ana.1032

8. Carmosino MJ, Brousseau KM, Arciniegas DB, Corboy JR. Initial evaluations for multiple sclerosis in a university multiple sclerosis center: outcomes and role of magnetic resonance imaging in referral. Arch Neurol. (2005) 62:585-90. doi: 10.1001/archneur.62.4.585

9. Miller DH, Weinshenker BG, Filippi M, Banwell BL, Cohen JA, Freedman MS, et al. Differential diagnosis of suspected multiple sclerosis: a consensus approach. Mult Scler. (2008) 14:1157-74. doi: 10.1177/1352458508096878

10. Boissy AR, Ford PJ. A touch of MS: therapeutic mislabeling. Neurology. (2012) 78:1981-5. doi: 10.1212/WNL.0b013e318259e0ec

11. Min JH, Kim BJ, Lee KH. Development of extensive brain lesions following fingolimod (FTY720) treatment in a patient with neuromyelitis optica spectrum disorder. Mult Scler. (2012) 18:113-5. doi: $10.1177 / 1352458511431973$

12. Cortese R, Magnollay L, Tur C, Abdel-Aziz K, Jacob A, De Angelis $\mathrm{F}$, et al. Value of the central vein sign at $3 \mathrm{~T}$ to differentiate MS
Diagnostic certainty must be established before starting longterm, expensive disease-modifying treatment. Reliable diagnosis of MS or of an alternative disorder requires application of clinical judgment, and correct interpretation of patient history, physical examination, imaging results, and laboratory findings, as well as clinician with MS expertise. Sometimes, a correct diagnosis may require periodic open-minded reassessments. Efforts should be made to strike a balance between timely MS diagnosis, and avoiding misdiagnosis. Reporting MS misdiagnosis may help prevent future diagnostic errors.

\section{AUTHOR CONTRIBUTIONS}

MG designed the article, wrote the manuscript, approved the version to be published, and agreed for all aspects of the work. JC, critically reviewed for intellectual content, approved the version to be published, and agreed for all aspects of the work.

\section{FUNDING}

This study was supported by funding from FLENI. from seropositive NMOSD. Neurology. (2018) 90:e1183-e1190. doi: 10.1212/WNL.0000000000005256

13. Maggi P, Absinta M, Grammatico M, Vuolo L, Emmi G, Carlucci G, et al. Central vein sign differentiates Multiple Sclerosis from central nervous system inflammatory vasculopathies. Ann Neurol. (2018) 83:283-94. doi: 10.1002/ana.25146

14. Freedman MS, Thompson EJ, Deisenhammer F, Giovannoni G, Grimsley G, Keir G, et al. Recommended standard of cerebrospinal fluid analysis in the diagnosis of multiple sclerosis: a consensus statement. Arch Neurol. (2005) 62:865-70. doi: 10.1001/archneur.62.6.865

15. Jarius S, Paul F, Franciotta D, Ruprecht K, Ringelstein M, Bergamaschi R, et al. Cerebrospinal fluid findings in aquaporin- 4 antibody positive neuromyelitis optica: results from 211 lumbar punctures. J Neurol Sci. (2011) 306:82-90. doi: 10.1016/j.jns.2011.03.038

16. Jarius S, Ruprecht K, Kleiter I, Borisow N, Asgari N, Pitarokoili K, et al. MOGIgG in NMO and related disorders: a multicenter study of 50 patients. Part 2: epidemiology, clinical presentation, radiological and laboratory features, treatment responses, and long-term outcome. J Neuroinflamm. (2016) 13:280. doi: 10.1186/s12974-016-0718-0

17. Lennon VA, Kryzer TJ, Pittock SJ, Verkman AS, Hinson SR. IgG marker of optic-spinal multiple sclerosis binds to the aquaporin-4 water channel. J Exp Med. (2005) 202:473-7. doi: 10.1084/jem.20050304

18. Kaneko K, Sato DK, Nakashima I, Nishiyama S, Tanaka S, Marignier R, et al. Myelin injury without astrocytopathy in neuroinflammatory disorders with MOG antibodies. J Neurol Neurosurg Psychiatry. (2016) 87:1257-9. doi: 10.1136/jnnp-2015-312676

19. Masuda S, Mori M, Arai K, Uzawa A, Muto M, Uchida T, et al. Epstein-Barr virus persistence and reactivation in neuromyelitis optica. J Neurol Neurosurg Psychiatry. (2015) 86:1137-42. doi: 10.1136/jnnp-2014-308095

20. Kakalacheva K, Regenass S, Wiesmayr S, Azzi T, Berger C, Dale RC, et al. Infectious mononucleosis triggers generation of IgG auto-antibodies against native myelin oligodendrocyte glycoprotein. Viruses. (2016) 8:E51. doi: 10.3390/v8020051

21. Jarius S, Eichhorn P, Franciotta D, Petereit HF, Akman-Demir G, Wick M, et al. The MRZ reaction as a highly specific marker of multiple sclerosis: reevaluation and structured review of the literature. J Neurol. (2017) 264:453-66. doi: 10.1007/s00415-016-8360-4

22. Dobson R, Ramagopalan S, Davis A, Giovannoni G. Cerebrospinal fluid oligoclonal bands in multiple sclerosis and clinically isolated syndromes: 
a meta-analysis of prevalence, prognosis and effect of latitude. $J$ Neurol Neurosurg Psychiatry. (2013) 84:909-14. doi: 10.1136/jnnp-2012-304695

23. Rovira A, Wattjes MP, Tintore M, Tur C, Yousry TA, Sormani MP, et al. Evidence-based guidelines: MAGNIMS consensus guidelines on the use of MRI in multiple sclerosis-clinical implementation in the diagnostic process. Nat Rev Neurol. (2015) 11:471-82. doi: 10.1038/nrneurol. 2015.106

24. Lennon VA, Wingerchuk DM, Kryzer TJ, Pittock SJ, Lucchinetti CF, Fujihara K, et al. A serum autoantibody marker of neuromyelitis optica: distinction from multiple sclerosis. Lancet. (2004) 364:2106-12. doi: 10.1016/S0140-6736(04)17551-X

25. Thompson AJ, Baranzini, SE, Geurts J, Hemmer B, Ciccarelli O. Multiple sclerosis. Lancet. (2018) 391:1622-36. doi: 10.1016/S0140-6736(18)30481-1

26. Brecht I, Weissbrich B, Braun J, Toyka KV, Weishaupt A, Buttmann M. Intrathecal, polyspecific antiviral immune response in oligoclonal band negative multiple sclerosis. PLoS ONE. (2012) 7:e40431. doi: 10.1371/journal.pone.0040431

27. Munger KL, Levin LI, O’Reilly EJ, Falk KI, Ascherio A. Anti-Epstein-Barr virus antibodies as serological markers of multiple sclerosis: a prospective study among United States military personnel. Mult Scler. (2011) 17:1185-93. doi: $10.1177 / 1352458511408991$

28. Pakpoor J, Disanto G, Gerber JE, Dobson R, Meier UC, Giovannoni G, et al. The risk of developing multiple sclerosis in individuals seronegative for Epstein-Barr virus: a meta-analysis. Mult Scler. (2013) 19:162-6. doi: $10.1177 / 1352458512449682$

29. Deuschle K, Hofmann J, Otto C, Bellmann-Strobl J, Scherner O, Klumbies $\mathrm{K}$, et al. Are there Epstein-Barr virus seronegative patients with multiple sclerosis? Mult Scler. (2013) 19:1242-3. doi: 10.1177/135245 8512472751

30. Pakpoor J, Ramagopalan SV. Epstein-Barr virus is a necessary causative agent in the pathogenesis of multiple sclerosis: yes. Mult Scler. (2013) 19:1690-1. doi: $10.1177 / 1352458513506505$

Conflict of Interest Statement: MG has received reimbursement for developing educational presentations from Merck-Serono Argentina, Genzyme Argentina and Novartis Argentina, and Biogen-Idec Argentina, and travel/accommodation stipends from Biogen-Idec Argentina, Teva-Tuteur Argentina, Merck-Serono Argentina, and Novartis Argentina. JC is a board member of Merck-Serono Argentina, Biogen-Idec LATAM, and Merck-Serono LATAM, and Genzyme global. JC is a board member of Merck-Serono Argentina, Novartis Argentina, Genzyme LATAM, Genzyme global, Biogen-Idec LATAM, and Merck-Serono LATAM. He is part of the Steering Committee for the clinical trials of Ofatumumab (Novartis Global). JC has received reimbursement for developing educational presentations for Merck-Serono Argentina, Merck-Serono LATAM, Biogen-Idec Argentina, Genzyme Argentina, Novartis Argentina, Novartis LATAM, Novartis Global, and TEVA Argentina as well as professional travel/accommodations stipends.

Copyright (๑) 2019 Gaitán and Correale. This is an open-access article distributed under the terms of the Creative Commons Attribution License (CC BY). The use, distribution or reproduction in other forums is permitted, provided the original author(s) and the copyright owner(s) are credited and that the original publication in this journal is cited, in accordance with accepted academic practice. No use, distribution or reproduction is permitted which does not comply with these terms. 